\title{
Computer Navigated Medial Opening Wedge High Tibial Osteotomy- Review of Literature
}

\author{
Kunal Dhurve* \\ Sydney Orthopaedic Research Institute, Australia \\ *Corresponding author: Kunal Dhurve, Sydney Orthopaedic Research Institute, L1, 445 Victoria Avenue, Chatswood, NSW 2067, Australia
}

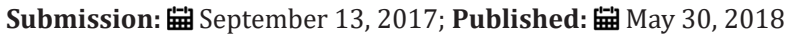

\section{Introduction}

Surgical management of arthritis of the knee in young and active patients has always been a challenge. Partial or total knee replacement is not the ideal surgical option due to lower implant survivorship in younger patients [1,2]. Hence the surgical management revolves around preserving the native joint with the aim of reduction of pain and improvement of function. The best example of a joint preservation procedure for knee arthritis is the high tibial osteotomy (HTO).

Correction of limb alignment with an osteotomy of the proximal tibia was first described by Jackson \& Waugh [3]. HTO works on the principle of unloading the arthritic cartilage by shifting the weight bearing axis to healthy joint compartment. HTO is best suited for patients with isolated medial compartment arthritis with pre-existing varus limb alignment and a relatively healthy lateral compartment. HTO is also used as unloading procedure concomitant to cartilage restoration surgery. Additionally, HTO is indicated in patients with multiligamentous knee injury with preexisting varus deformity.

Two principle techniques for $\mathrm{HTO}$ are the lateral closing wedge and the medial opening wedge osteotomy (MOWHTO). The lateral closing wedge osteotomy was popularised by Coventry [4]. The advantages of lateral closing wedge osteotomy is the lack of need for bone grafting after the osteotomy, earlier weight bearing and faster healing of the osteotomy. The disadvantages with a lateral closing wedge osteotomy includes the risk of injuring the common peroneal nerve [5], the need for fibular osteotomy and offset of the proximal tibia when large correction is performed which can make subsequent arthroplasty technically difficult [6] .

Hernigou et al. [7] introduced the technique of medial opening wedge osteotomy in 1987. The advantages of a medial opening wedge osteotomy include the ease of gradual intra operative correction of the limb alignment in addition to avoiding the aforementioned complications of lateral closing wedge osteotomy. However, the disadvantages with a medial opening wedge osteotomy is the need for bone grafting, delayed weight bearing till radiological evidence of healing ,potential for fracture of the lateral tibial plateau [8], non- union [9] and the chances of loss of correction. The initial stability of the osteotomy can be improved with the use of locking plates for fixation. The availability of bone substitutes has helped in circumventing the problems associated with autologous graft harvesting. Medial opening wedge osteotomy has gained popularity over the recent years due to the ease of intraoperative correction and lesser serious complication as compared to the lateral closing wedge osteotomy.

Medial opening wedge osteotomy is a technically demanding procedure as the results of this surgery are largely dependent on the accuracy of intra operative correction of the limb alignment $[10,11]$. The aim is to transfer the weight bearing axis to the lateral compartment with slight over correction as it has been shown that over correction to valgus angle is associated with better outcomes in terms of symptom relief and long term survival of surgery $[7,10,12]$.This implies precise pre-operative planning and accurate determination of correction of alignment intra operatively. Preoperative planning involves assessing long leg films for measuring limb alignment and calculation of the wedge of bone that needs to be removed during the osteotomy. The magnification of radiographs needs to be taken into account while calculating the wedge size. The biggest challenge is the practical application of the planning during surgery.

Traditionally, limb alignment has been assessed intra operatively with long rods or diathermy cable using fluoroscopy. Krettek et al. [13] described using the diathermy cable for assessment of limb alignment. The cable is centered over the center of femoral head and the center of the ankle joint under fluoroscopy guidance which represents the mechanical axis of the lower limb. The correction is deemed to be achieved if the mechanical axis passes through a point $30 \%$ to $40 \%$ of the width of the lateral tibial plateau. However, the accuracy of this technique is dependent on the limb rotation, parallax and quality of images. Additionally this technique provides only momentary assessment of limb alignment that too only in the frontal plane. 
As computer navigation gained popularity, experiments were undertaken in cadavers to test its application in HTO. Hankemeier et al. [14] compared the accuracy of intra operative analysis of mechanical axis by cable method with navigation in cadaveric lower limbs. The measurement of mechanical axis by cable method and navigation system was compared with CT scan based measurement of the mechanical axis. The difference in the measurement by cable method and CT based measurement was $6.0 \pm 3.1 \%$ whereas the difference was $2.6 \pm 1.8 \%$ for navigation system clearly showing that navigation system based measurements were more accurate. This work was further extended by Hankemeier et al. [15] by comparing the accuracy of HTO by conventional and navigation system in cadaveric knees. They report a significantly lower variability in correction of the mechanical axis and fluoroscopy radiation time with the navigated HTO as compared to conventional HTO using cable method for assessment of mechanical axis. Lutzner et al. [16] randomly assigned 19 cadaveric legs to conventional and navigated HTO. They aimed at shifting the weight bearing line to $80 \%$ of the width of the tibia. The mean deviation from the targeted $80 \%$ width was $1 \%$ for navigated HTO and $8.6 \%$ for conventional HTO. The authors concluded that navigated HTO achieves better correction of weight bearing line that conventional HTO in cadaveric legs. The favourable outcomes of cadaveric studies encouraged further clinical studies for assessing the accuracy and reproducibility of HTO using computer navigation.

Saragaglia \& Robert [17] compared 28 navigated open wedge HTOs with 28 conventional HTOs. They reported a reproducibility of $96 \%$ in achieving a mechanical axis of $184 \pm 2$ degrees when HTO was performed using navigation as compared to reproducibility of $71 \%$ with conventional HTO. Additionally, they showed a high degree of accuracy when navigation was used for double level osteotomy. Maurer \& Wassmer [18] compared the accuracy of correction between 23 patients with conventional HTO and 44 patients with navigated HTO. They could achieve a satisfactory alignment of $182-186$ degrees in $73 \%$ of patients with navigated HTO and in 30\% with conventional HTO. They also found a good correlation between preoperative varus deformity measured on $\mathrm{X}$ rays with the angle of deformity measured by the navigation system. The final correction angle achieved with navigation correlated with the angle measured on post-operative long leg X rays.

The main focus of these studies has been the assessment of accuracy of correction in the coronal plane as the navigation systems did not allow for monitoring the changes in the tibia slope in the sagittal plane. There is a risk of increasing the tibial slope with a MOWHTO [19]. Alterations in tibial slope have been shown to influence knee kinematics and joint contact pressure [20-22]. Researchers devised several techniques to overcome this shortcoming of early navigation systems. Noyes et al. [23] performed a 3-dimensional analysis of the proximal tibia and introduced the three triangle method of correction of axial alignment and tibial slope during a medial opening HTO. They suggested that the anterior osteotomy gap at the level of tibial tubercle should be one half of the postero-medial gap in order to maintain the native tibial slope. A gap error of $1 \mathrm{~mm}$ at the tibial tubercle could result in a change of posterior tibial slope of 2 degrees.

Kim et al. [24] monitored the tibial slope by inserting two guide wires such that they overlapped each other when the fluoroscope was tilted caudally by 10 degrees to represent the proximal tibial slope. As an additional measure to control the slope, they ensured that the width of the osteotomy at the postero- medial corner was twice as that at the anteromedial corner. They applied this technique to patients undergoing conventional and navigated HTO and reported better radiological results with navigated HTO for accuracy of correction and reproducibility. On clinical assessment, navigated group had higher Hospital for Special Surgery knee score and Lysholm knee scores as compared to the conventional group. There was no significant alteration in the tibial slope in both groups.

Song et al. [25] reported on 40 patients treated with navigated MOWHTO. All patients had a preoperative CT scan which was used for construction a 3D surface model. Three dimensional virtual HTO was performed while maintaining the native tibial slope. They found that a ratio of 0.67 between the anterior and posterior opening gaps was needed to maintain the native slope of tibia. They reported satisfactory outcomes with fewer outliers in terms of mechanical axis. The posterior tibial slope was well maintained post operatively. In order to maintain the posterior tibial slope they recommended that the osteotomy should be parallel to joint line in the sagittal plane, posterior corticotomy should be complete, posterior soft tissues should be released adequately, the osteotomy should be fixed with two plates with the posterior plate placed as posterior as possible and the post-operative knee extension should be maintained to the same degrees as preoperative values during navigation.

The navigation technology has evolved over the years and the current navigation systems are equipped to provide sagittal plane parameters in addition to the coronal plane parameters. Computer navigation systems are now available in two dimensional (2-D) and three dimensional (3-D) versions. Iorio et al. [26] prospectively followed up 24 patients (27 knees) who were randomly assigned to conventional HTO (11 patients) and navigated HTO (13 patients) with a median follow up of 39 months. They used the OrthopilotTM 3D (Braun Aesculap, Tuttlingen, Germany) navigation system for navigated HTO. An additional transmitter was fixed to proximal tibia in six patients to monitor the changes in tibial slope during HTO. The American Knee Society Score and the Modified Cincinnati Rating System Questionnaire were used for clinical assessment. There was no statistically significant difference in between the two groups on clinical assessment. The navigation group showed a reproducibility of $85 \%$ in achieving a mechanical axis of $182-186$ degrees. Additionally, the modification in the tibial slope within the targeted \pm 2 degrees was achieved in all patients who underwent navigated HTO. This study showed that 3 dimensional navigation systems can help in monitoring and maintain the native tibial slope during an HTO. 
Yim et al. [27] compared the accuracy of 2-D navigation with 3-D navigation in maintaining the posterior tibial slope in MWHTO. Seventeen patients were assigned to 2-D navigation (group A) and 17 patients to 3 -D navigation (group B). The mechanical axis was corrected to a mean valgus of 2.81 degrees in group A and 3.15 degrees in group $B(p=0.442)$. There was significant difference in the change in the posterior slope after HTO in group A when compared with preoperative tibia slope. They suggested that using three dimensional computer navigation for HTO can help in maintaining the posterior tibial slope.

\section{Complications of Medial Opening Wedge High Tibial Osteotomy}

Although MOWHTO is associated with fewer complications as compared to a lateral closing wedge osteotomy, complications such as fractures, non-union/ delayed union, loss of reduction and implant prominence can occur.

\section{Fractures}

MOWHTO involves creation of a partial osteotomy while maintaining the integrity of the lateral cortex. This lateral cortex serves as a hinge on which the osteotomy is opened to achieve correction of limb alignment. However, fractures of the lateral cortex during a medial opening wedge osteotomy have been reported with an incidence ranging from $2.2 \%$ to $9 \%$ [28-31]. The implications of the fracture are that it can lead to loss of correction and translation of the osteotomy and may require stabilisation from the lateral aspect. The lateral cortical fracture can be avoided by stopping the osteotomy $10 \mathrm{~mm}$ short of lateral cortex and gradually distracting the osteotomy to allow for plastic deformation of the lateral cortex.

The osteotomy can propagate proximally and can lead to an intra-articular fracture of the lateral tibial plateau. The reported incidence of lateral plateau fracture in literature range from $6.7 \%$ to $8.2 \%[8,28]$. This usually occurs either when the depth of osteotomy is inadequate or when the osteotomy ends closer to the joint surface than the lateral cortex of tibial. In these situations, the osteotomy travels the path of least resistance and opens into the lateral compartment of the knee. The lateral tibial plateau fractures needs fixation with cancellous screws from the lateral side.

\section{Delayed union and non-union}

It was initially believed that MOWHTO is associated with higher chances of delayed and non-union as compared to lateral closing wedge osteotomy [32,33]. However, recent studies show that the rates of non-union rate similar for opening and closing wedge osteotomies [34-36]. Factors associated with non-union include smoking, higher BMI and lateral cortical fracture [37]. Strategies for prevention of non-union include proper patient selection, stable fixation of osteotomy and reinforcing weight bearing restrictions.

\section{Loss of correction}

The factors associated with loss of correction after MOWHTO include type of fixation and BMI of the patient [38]. Loss of correction was higher with first generation puddu plates [8]. The chances of loss of correction have reduced with the use of locking plates such as the Tomofix [34].

\section{Complications unique to navigation}

Complications associated with the use of navigation include additional incisions for insertion of half pins, possibility of iatrogenic fracture around the pins, vascular injury during insertion of pins, line of site issues, slightly increased surgical time and increased economic burden.

\section{Conclusion}

The technique of medial opening wedge osteotomy has evolved considerably over the years. The addition of computer navigation to MOWHTO has helped in improving the accuracy and reproducibility of correction of alignment. The long term influence of improved accuracy of correction of alignment on the results of HTO remains to be seen.

\section{References}

1. Gioe TJ, Novak C, Sinner P, Ma W, Mehle (2007) Knee arthroplasty in the young patient: survival in a community registry. Clin Orthop Relat Res 464: 83-87.

2. Rand JA, Trousdale RT, Ilstrup DM, Harmsen W (2003) Factors affecting the durability of primary total knee prostheses. The JJ Bone Joint Surg Am 85-A(2): 259-265.

3. Jackson JP, Waugh W (1961) Tibial osteotomy for osteoarthritis of the knee. Journal of Bone \& Joint Surgery 43-B(4):746-751.

4. Coventry MB (1965) Osteotomy of the upper portion of the tibia for degenerative arthritis of the knee. The Journal of Bone \&Amp 47(5): 984-990.

5. Bauer T, Hardy P, Lemoine J, Finlayson DF, Tranier S, et al. (2005) Drop foot after high tibial osteotomy: a prospective study of aetiological factors. Knee Surg Sports Traumatol Arthrosc 13(1): 23-33.

6. Cerciello S, Vasso M, Maffulli N, Neyret P, Corona K, et al. (2014) Total knee arthroplasty after high tibial osteotomy. Orthopedics 37(3):191198.

7. Hernigou P, Medevielle D, Debeyre J, Goutallier D (1987) Proximal tibial osteotomy for osteoarthritis with varus deformity. a ten to thirteen-year follow-up study. J Bone Joint Surg Am 69(3): 332-354.

8. Spahn G (2004) Complications in high tibial (medial opening wedge) osteotomy. Arch Orthop Trauma Surg 124(10): 649-653.

9. Warden SJ, Morris HG, Crossley KM, Brukner PD, Bennell KL (2005) Delayed- and non-union following opening wedge high tibial osteotomy: surgeons' results from 182 completed cases. Knee Surg Sports Traumatol Arthrosc 13(1): 34-37.

10. Sprenger TR, Doerzbacher JF (2003) Tibial osteotomy for the treatment of varus gonarthrosis survival and failure analysis to twenty-two years. Bone Joint Surg Am 85(3): 469-474.

11. Hernigou P, Medevielle D, Debeyre J, Goutallier D (1987) Proximal tibial osteotomy for osteoarthritis with varus deformity. a ten to thirteen-year follow-up study. J Bone Joint Surg Am 69(3): 332-354.

12. Aglietti P, Buzzi R, Vena LM, Baldini A, Mondaini A (2003) High tibial valgus osteotomy for medial gonarthrosis: a 10- to 21-year study. J Knee Surg 16(1): 21-26.

13. Krettek C, Miclau T, Grün O, Schandelmaier P, Tscherne H (1998) 
Intraoperative control of axes, rotation and length in femoral and tibial fractures technical note. Injury 29(Supp 3): 29-39.

14. Hankemeier S, Hufner T, Wang G, Kendoff D, Zheng G, et al. (2005) Navigated intraoperative analysis of lower limb alignment. Arch Orthop Trauma Surg 125(8): 531-535.

15. Hankemeier S, Hufner T, Wang G, Kendoff D, Zeichen J, et al. (2006) Navigated open-wedge high tibial osteotomy: advantages and disadvantages compared to the conventional technique in a cadaver study. Knee Surg Sports Traumatol Arthrosc 14(10): 917-921.

16. Lutzner J, Gross AF, Gunther KP, Kirschner S (2010) Precision of navigated and conventional open-wedge high tibial osteotomy in a cadaver study. Eur J Med Res 15(3): 117-120.

17. Saragaglia DMD, Roberts J (2005) Navigated osteotomies around the knee in 170 patients with osteoarthritis secondary to genu varum. Orthopedics 28(supp 10): 1269-1274.

18. Maurer F, Wassmer G (2006) High tibial osteotomy: does navigation improve results? Orthopedics 29(Supp 10): 130-132.

19. Lustig S, Scholes CJ, Costa AJ, Coolican MJ, Parker DA (2013) Different changes in slope between the medial and lateral tibial plateau after open-wedge high tibial osteotomy. Knee Surg Sports Traumatol Arthrosc 21(1): 32-38.

20. Giffin JR, Vogrin TM, Zantop T, Woo SLY, Harner CD (2004) Effects of increasing tibial slope on the biomechanics of the knee. Am J Sports Med 32(2): 376-382.

21. Rodner CM, Adams DJ, Doran VD, Tate JP, Santangelo SA, et al. (2006) Medial opening wedge tibial osteotomy and the sagittal plane: the effect of increasing tibial slope on tibiofemoral contact pressure. Am J Sports Med 34(9): 1431-1441.

22. Agneskirchner JD, Hurschler C, Colsman C, Imhoff AB, Lobenhoffer $P$ (2004) Effect of high tibial flexion osteotomy on cartilage pressure and joint kinematics: a biomechanical study in human cadaveric knees: winner of the aga-donjoy award 2004. Arch Orthop Trauma Surg 124(9): 575-584.

23. Noyes FR, Goebel SX, West J (2005) Opening wedge tibial osteotomy: The 3-triangle method to correct axial alignment and tibial slope. Am J Sports Med 33(3): 378-387.

24. Kim SJ, Koh YG, Chun YM, Kim YC, Park YS (2009) Medial opening wedge high-tibial osteotomy using a kinematic navigation system versus a conventional method: a 1-year retrospective, comparative study. Knee Surg Sports Traumatol Arthrosc 17(2):128-134.

25. Song EK, Seon JK, Park SJ (2007) How to avoid unintended increase of posterior slope in navigation-assisted open-wedge high tibial osteotomy. Orthopedics 30(10 supp): 127-131.

26. Iorio R, Pagnottelli M, Vadala A, Giannetti S, Di Sette P (2013) Open- wedge high tibial osteotomy: comparison between manual and computer-assisted techniques. Knee Surg Sports Traumatol Arthrosc 21(1): 113-119.

27. Yim JH, Seon JK, Song EK (2012) Posterior tibial slope in medial openingwedge high tibial osteotomy: 2-d versus 3 -d navigation. Orthopedics 35(10 supp): 60-63.

28. Chae DJ, Shetty GM, Wang KH, Montalban AS, Kim JI (2011) Early complications of medial opening wedge high tibial osteotomy using autologous tricortical iliac bone graft and t-plate fixation. Knee 18(4): 278-284.

29. Esenkaya I, Elmali N (2006) Proximal tibia medial open-wedge osteotomy using plates with wedges: early results in 58 cases. Knee Surg Sports Traumatol Arthrosc 14(10): 955-961.

30. Nelissen EM, Langelaan EJ, Nelissen RG (2010) Stability of medial opening wedge high tibial osteotomy: a failure analysis. Int Orthop 34(2): 217-223.

31. Song EK, Seon JK, Park SJ, Jeong MS (2010) The complications of high tibial osteotomy: closing- versus opening-wedge methods. J Bone Joint Surg Br 92(9): 1245-1252.

32. Bekerom MP, Patt TW, Kleinhout MY, Vis HM, Albers GH (2008) Early complications after high tibial osteotomy: a comparison of two techniques. J Knee Surg 21(1): 68-74.

33. Warden SJ, Morris HG, Crossley KM, Brukner PD, Bennell KL (2005) Delayed- and non-union following opening wedge high tibial osteotomy: surgeons' results from 182 completed cases. Knee Surg Sports Traumatol Arthrosc 13(1): 34-37.

34. Brosset T, Pasquier G, Migaud H, Gougeon F (2011) Opening wedge high tibial osteotomy performed without filling the defect but with locking plate fixation (tomofix) and early weight-bearing: prospective evaluation of bone union, precision and maintenance of correction in 51 cases. Orthop Traumatol Surg Res 97(7): 705-711.

35. El-Assal MA, Khalifa YE, Abdel-Hamid MM, Said HG, Bakr HM (2010) Opening-wedge high tibial osteotomy without bone graft. Knee Surg Sports Traumatol Arthrosc 18(7): 961-966.

36. Meidinger G, Imhoff AB, Paul J, Kirchhoff C, Sauerschnig M (2011) May smokers and overweight patients be treated with a medial open-wedge hto? risk factors for non-union. Knee Surg Sports Traumatol Arthrosc 19(3): 333-339.

37. van Houten AH, Heesterbeek PJ, van Heerwaarden RJ, van Tienen TG, Wymenga AB (2014) Medial open wedge high tibial osteotomy: can delayed or nonunion be predicted? Clin Orthop Relat Res 472(4): 12171223.

38. Miller BS, Downie B, McDonough EB, Wojtys EM (2009) Complications after medial opening wedge high tibial osteotomy. Arthroscopy 25(6): 639-646.
Creative Commons Attribution 4.0 International License

For possible submissions Click Here

\section{Submit Article}

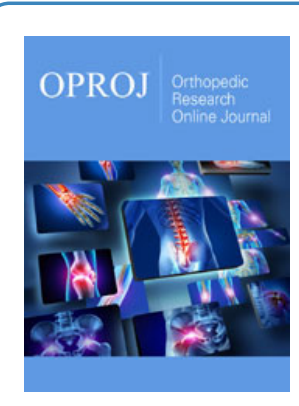

Orthopedic Research Online Journal

\section{Benefits of Publishing with us}

- High-level peer review and editorial services

- Freely accessible online immediately upon publication

- Authors retain the copyright to their work

- Licensing it under a Creative Commons license

- Visibility through different online platforms 